\title{
Comparative Analysis of Cone Resistances in Cone Penetration Test (CPTu) and Dynamic Probe Heavy Test (DPH)
}

\author{
Irena Bagińska $\mathbb{D}$
}

Received: 12 November 2018/Accepted: 13 May 2020/Published online: 20 May 2020

(C) The Author(s) 2020

\begin{abstract}
This paper presents a comparison of geotechnical soil testing with the use of Piezocone Penetration Test (CPTu) and Dynamic Probing Heavy $(\mathrm{DPH})$ in a uniform coarse-grained medium located in southwest part of Poland. The soil medium was identified in detail. The interpretation of results included both grain-size analysis of samples from drillholes and soil behavior type results obtained from the Robertson nomograph (Soil behaviour type from the CPT: an update. In: 2nd international symposium on cone penetration testing, USA, 2010). Cone penetration resistances $q_{c}$ obtained in the static CPTu tests were compared with the results of dynamic probing $\mathrm{N} \mathrm{O}_{H}$ and of dynamic point resistance $q_{d}$ obtained in the dynamic DPH tests. The evaluation of soil density index was based on both dynamic DPH and static CPTu probing. The obtained results were analyzed and plotted in a form of graphs corresponding to the Eurocode 7 and other works describing coarse-grained soils.
\end{abstract}

\section{Bagińska $(\bowtie)$}

Department of Geotechnology, Hydro Technology, and Underground and Hydro Engineering, Faculty of Civil

Engineering, Wroclaw University of Science and

Technology, 27 Wybrzeże Wyspiańskiego st.,

50-370 Wrocław, Poland

e-mail: irena.baginska@pwr.edu.pl
Keywords Static cone penetration test CPTu . Dynamic probing DPH $\cdot$ Cone resistance $q_{c} \cdot$ Dynamic point resistance $q_{d}$. Soil density index $I_{D}$

\section{Introduction}

Static CPTu (Piezocone Penetration Test) probing and dynamic DPH (Dynamic Probing Heavy) tests have been extensively used to evaluate geotechnical soil parameters. Both techniques allow determination of density index $\left(I_{D}\right)$, but typically they are not performed simultaneously, as they are assumed to provide comparable data on the compaction degree of noncohesive soils. Also, the DPH tests are believed to be unnecessary if the CPTu tests have been performed. This paper focuses on the presentation and comparison of both probing techniques, inter alia by identifying soil density index, which is the basic parameter measured in-situ for coarse soils during any construction project. This value may be determined with several dynamic probing techniques and with static probing. In the case of dynamic probing, the measurement and its interpretation is discrete, i.e.at every $10 \mathrm{~cm}$ or $20 \mathrm{~cm}$, while in the case of static probing with the use of an electrical cone, the measurement and its interpretation is almost continuous at every $2 \mathrm{~cm}$. This paper compares the interpretative results of 
soil density index obtained from DPH dynamic test with the results obtained from CPTu probing.

Additionally, the paper presents a comparative analysis of both the measured values $\left(q_{c}\right.$ for $\mathrm{CPTu}$ with $N 1 O_{H}$ for DPH) and the derivative values from both tests, with the aim to verify a thesis that correlation between the results from both tests is possible, safe and harmonized with the $\widehat{q}_{c}$ nomograph of the relationship between the $q_{c}$ values from CPTu and the $\mathrm{N} \mathrm{O}_{\mathrm{H}}$ measurements from DPH included in EN 1997-2:2007 Eurocode 7 Annex G.4 (after Stenzel and Melzer 1978).

Such investigations are of special importance for verifying local applicability of cone penetration resistance $\left(\widehat{q}_{c}\right)$ estimations in sands and sand-gravels on the basis of DPH, as represented in EN 19972:2007 Eurocode 7, Annex G.4. The resulting $\widehat{q}_{c}$ vs depth pattern may serve to establish the load-bearing capacity limits of piles on the basis of the correlations established from the test results of static load-bearing capacity of piles as per EN 1997-2:2007 Eurocode 7.

The Eurocode 7 is not the only source allowing direct correlation of the measurement values obtained from dynamic and static probing. Already in 1968, Melzer (1968) presented a correlation between DPH and CPT tests performed with a mechanical cone. Currently, an increasingly popular solution has become to perform penetration tests with the use of electrical cones (piezocones), whose $q_{c}$ measurement values are lower than in the case of mechanical cones (Bustamante and Gianeselli 1993). Therefore, it seems important to search for new correlations established for various local conditions and various non-cohesive soils (Czado and Pietras 2012; Lingwanda et al. 2015; Kodicherla and Nandyala 2016; dos Santos and Bicalho 2017). Research should also include finding the relationship between CPTu measurements and various dynamic probing techniques (Dynamic Penetrometer Light, Medium, super Heavy), as well as SPT (Standard Penetration Test) results, as indicated by Gadeikis et al. (2010); Pinheiro et al. (2018) and Ampadu et al. (2018) for non-cohesion soils and by Mahler \& Szendefy (2009) for cohesive soils.

The tests presented in this paper have been performed with electrical CPTu piezocone having an area of $10 \mathrm{~cm}^{2}$, as per EN ISO 22476-1:2012, while the DPH tests were performed with a heavy probe $(50 \mathrm{~kg}$ ) as per EN ISO 22476-2:2005. The influence of soil friction on the side surface of rods used in CPTu and DPH measurements was ignored in this work. The obtained results were analyzed and plotted in this paper in a form of graphs corresponding to Eurocode 7 and other, not only Polish, works in the field (Gadeikis et al. 2010; Czado and Pietras 2012).

The basic statistical measures used in this paper include:

Mean value of parameter $\hat{\mu}=\frac{1}{k} \sum_{i=1}^{k} X_{i}$;

Standard deviation $(\mathrm{SD}) \hat{\sigma}^{2}=\frac{1}{k-1} \sum_{i=1}^{k}\left(X_{i}-\hat{\mu}\right)^{2}$;

Coefficient of variation $(\mathrm{CV}) c_{V}=\frac{\hat{\sigma}}{\hat{\mu}}$,

where $X_{i}=$ the measured value of parameter; $k=$ the number of observations.

\section{Test site and results}

Morphologically, the test site is a part of the Central Polish Lowland. More specifically, it is located at the southwest border of the Dałków Hills being part of the Polkowice Hills. The shallowest formations are represented by sand-gravel deposits, clays and dusts classified as South-Polish, Central-Polish and NorthPolish Glaciations.

The tests covered an area of approx. $50 \mathrm{~m}^{2}$ and were conducted in an industrial space, which prevented a very large number of tests at regular distances. The tests included one static CPTu probing (Fig. 1), four dynamic PDH probings (Fig. 3) and four drillings in the DPH test axis in order to verify the type of soil and to collect soil samples for grain-size analysis. The test points were located at greater distances than the minima required in industry standards due to the disruptive influence of one test on another ( $>2 \mathrm{~m}$ as per EN ISO 22476-2:2005). The DPH probings were spaced at approx. $5 \mathrm{~m}$. The minimum distance between the DPH test points in relation to $\mathrm{CPTu}$ tests was approx. $3 \mathrm{~m}$, and the maximum distance- $10 \mathrm{~m}$.

The test site was located near a very large industrial object with spread foundations at $3.5 \mathrm{~m} \mathrm{b.g.l}$. Based on archived information, the foundations were identified to be located in a wide excavation, which was later filled with local soil. Therefore, the grain-size analysis was performed for 3 samples collected above and 7 samples collected below $3.5 \mathrm{~m}$ b.g.l. The grainsize distribution was measured with the use of the 
Fig. 1 Measurement of cone resistance $q_{c}$, local friction $f_{s}$ and pore-water pressure $u_{2}$ from CPTu

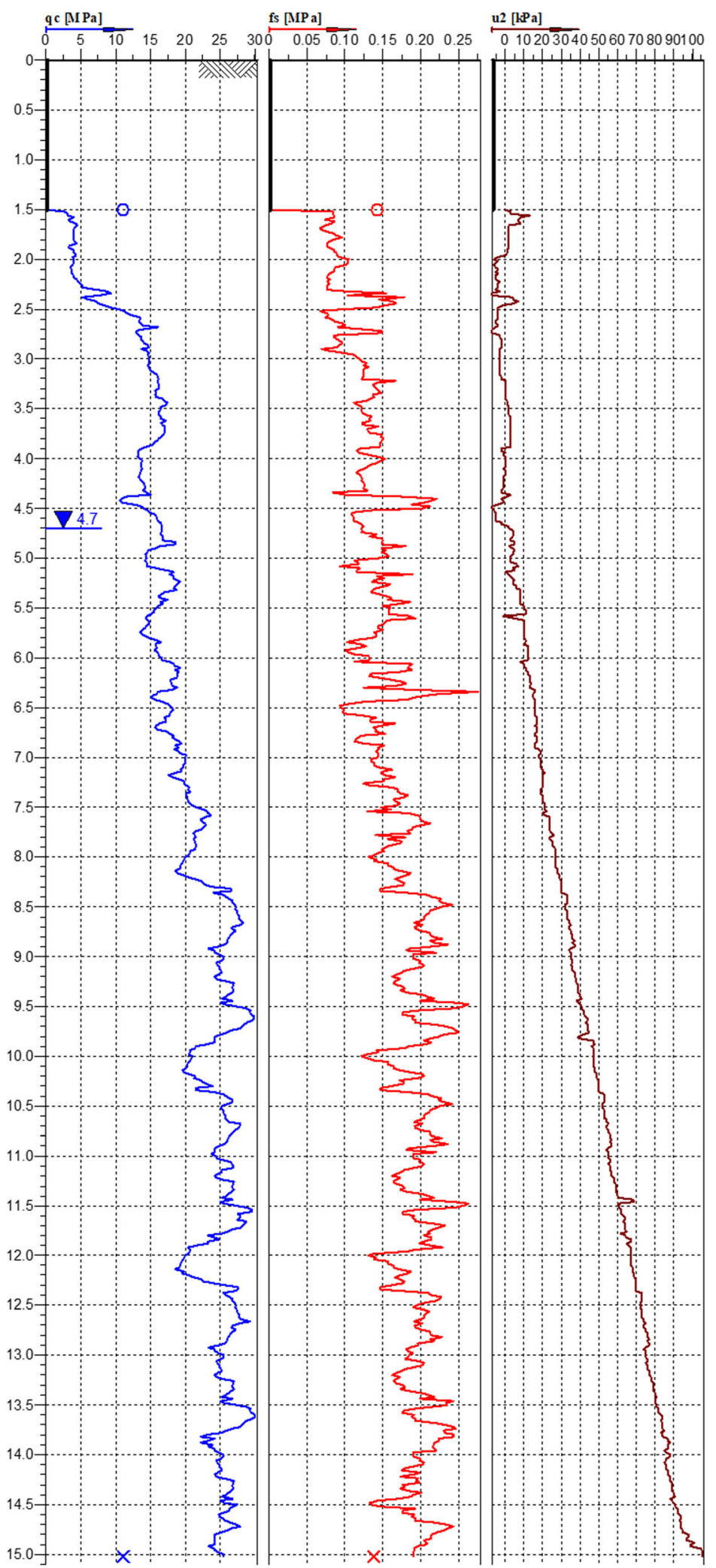


sieving method as per EN ISO 17892-4:2016. The samples showed the -dominance of sand gravel ( $\mathrm{grSa}$ ), and medium sand (MSa) at lower depths, as per EN ISO 14688-2:2018. Mean value of uniformity coefficient $C_{u}$ was 3.29, and of grain size distribution curve $C_{c}-1.15\left(C_{u}=\frac{d_{60}}{d_{10}}\right.$ and $C_{c}=\frac{d_{30}^{2}}{d_{10} \cdot 60^{2}}$, calculated in accordance with EN ISO 14688-1:2018). Based on the obtained results, the soil was classified as poorlygraded sand (SP as per ISO 22476-2:2005). The drilling operations revealed a free groundwater table at $4.7 \mathrm{~m}$ b.g.l., which was allowed for in the interpretations of both CPTu and DPH tests.

As shown in Fig. 2, the evaluation of the soil type based on CPTu was made on the basis of the SBT (Soil Behavior Type) soil classification, Robertson (2010), as SBT6 (Sands: clean sand to silty sands). Each of the points indicated in the nomograph corresponds to mean current values measured per each $10 \mathrm{~cm}$ in the quasi continuous $\mathrm{CPTu}$ measurement every $2 \mathrm{~cm}$, from 2.5 to $15.0 \mathrm{~m} \mathrm{b.g.l}$. The majority of the measurement points forms a dense group, indicating a significant uniformity of the tested soil medium.

Figure 3a presents the recorded $N^{\prime} 10_{H}$ for all four DPH tests versus depth without consideration to increased number of blows below the groundwater table, while Fig. $3 \mathrm{~b}$ shows the $N^{\prime} 10_{\text {Hmean }}$ pattern before and the $N 10_{\text {Hmean }}$ pattern after allowing for the influence of groundwater per each $10 \mathrm{~cm}$ of the DPH test penetration. The influence of water on the measurement was allowed for in accordance with the recommendations of EN ISO 22476-2:2005, i.e. in accordance with relationship (1) below $4.7 \mathrm{~m}$ b.g.l.

$N 10_{H}=a_{1} N^{\prime} 10_{H}+a_{2}$

Values $a_{1}$ and $a_{2}$ in (1) have been assumed equal to 1.3 and 2.0, respectively, as is the case for poorly graded sands (SP) having $C_{u} \leq 3$, as mean $C_{u}$ is 3.29 and is significantly smaller than 6 .
Fig. 2 Identification of soil type according to the Robertson (2010) classification, where $p_{a \_ \text {atmospheric pressure }}$ ( $\sim 100 \mathrm{kPa}), R_{f}$-friction ratio $R_{f}=\frac{f_{s}}{q_{c}} \cdot 100 \%$

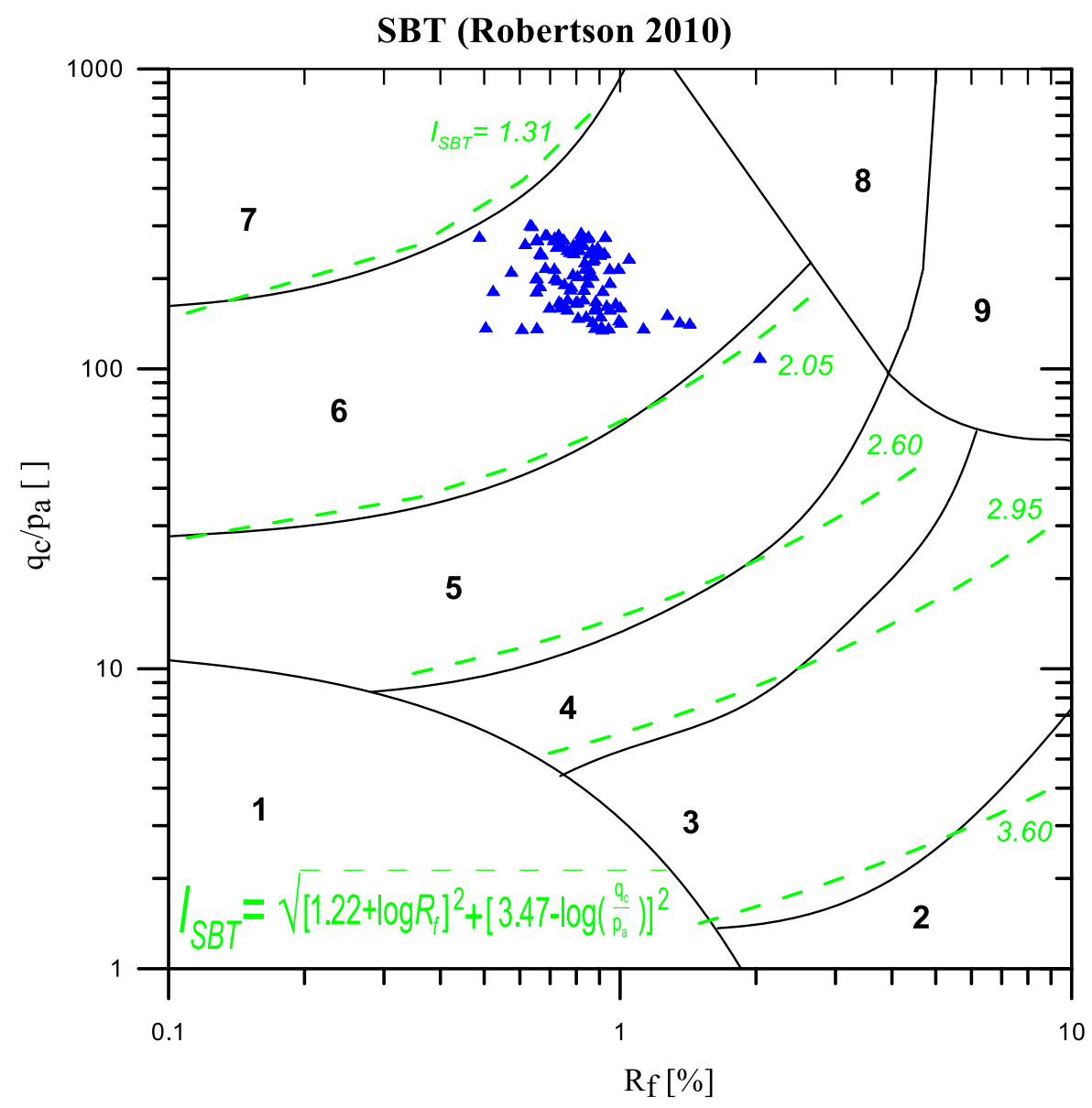




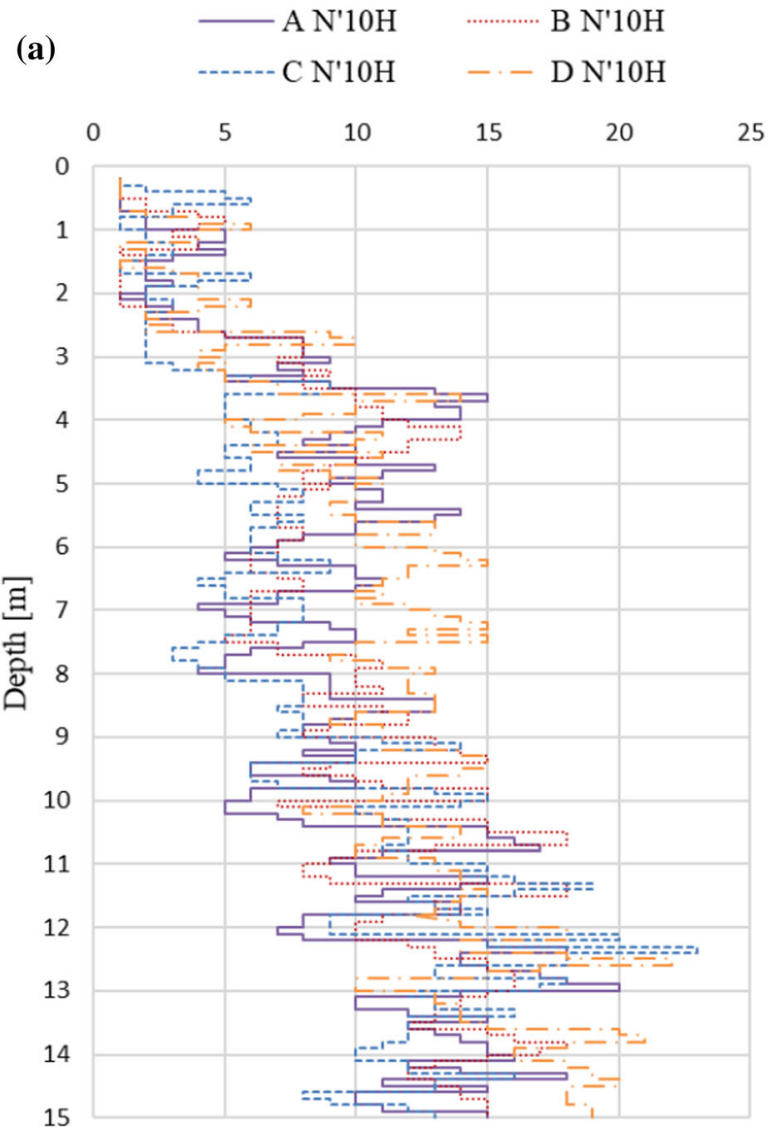

(b)

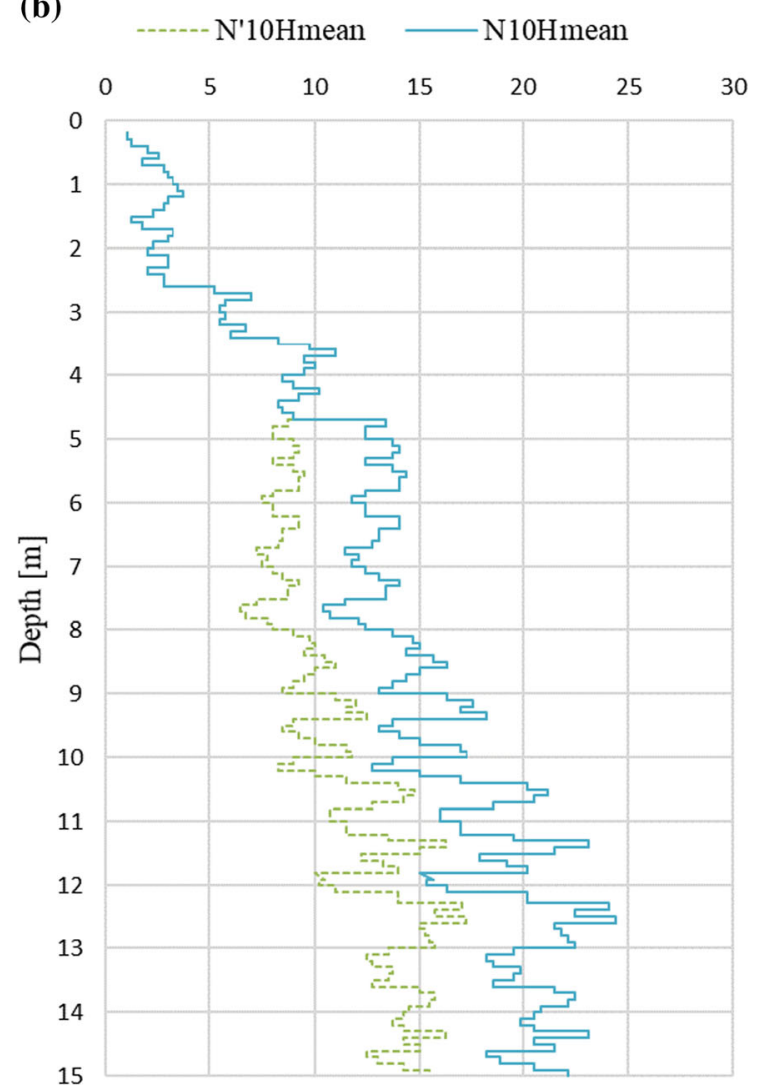

Fig. 3 a Pattern of the $N^{\prime} 10_{H}$ recorded in four DPH measurements; $\mathbf{b}$ Mean DPH test measurement value without $\left(N^{\prime} 10_{H m e a n}\right)$ and with $\left(N 10_{\text {Hmean }}\right)$ allowance for the influence ground water below $4.7 \mathrm{~m}$ b.g.l., in accordance with Eq. (1)

The evaluation of soil density index was based on both dynamic DPH and static CPTu probing. Calculations of the density index based on the DPH test were based on the most commonly employed Eurocode 7 formula for poorly graded sands (SP) (2).

$I_{D D P H}=0.38 \cdot \log N 10_{\text {Hmean }}+0.23$

In (2) $N 10_{\text {Hmean }}$ is the number of free hammer falls per each $10 \mathrm{~cm}$ of the DPH probe head penetration, with allowance for an increased number of falls below the groundwater table level, in accordance with relationship (1) (Fig. 3b).

In the case when $\mathrm{I}_{\mathrm{D}}$ (soil density index) is identified from the CPTu test, a number of correlations can be used, which should be verified (Bałachowski 2006; Jamiolkowski et al. 2003). In this work, two such correlations were used, in accordance with Lunne et al. (1997) Lancellotta expression (3) and in accordance with Jamiolkowski et al. (2003) (4).

$$
\begin{aligned}
& I_{D C P T u}=-98+66 \cdot \log \left(\frac{q_{c}}{\left(\sigma_{v o}^{\prime}\right)^{0.50}}\right)[\%] \\
& I_{D C P T u}=-1.292+0.268 \cdot \ln \left(\frac{q_{c}}{\left(\sigma_{v o}^{\prime}\right)^{0.52}}\right)[-]
\end{aligned}
$$

where: $q_{c}$-cone resistance $[\mathrm{MPa}], \sigma_{v o}^{\prime}$-vertical component of effective stress [MPa].

Figure 4 shows the values of the $I_{D}$ soil density index, calculated against depth in accordance with Eqs. (2), (3) and (4). Down to $4.7 \mathrm{~m}$ b.g.l., the density increases with depth. Below $4.7 \mathrm{~m}$ b.g.l., the density becomes stable in the range from 0.65 to 0.85 , allowing the classification of soil as dense (look at Fig. 4). Coarse-grained soil grSa/FSa may be eventually classified to be dense soil, and locally to be between dense and very dense (EN/ISO 14688-2:2018). Table 1 shows the calculated $I_{D}$ 


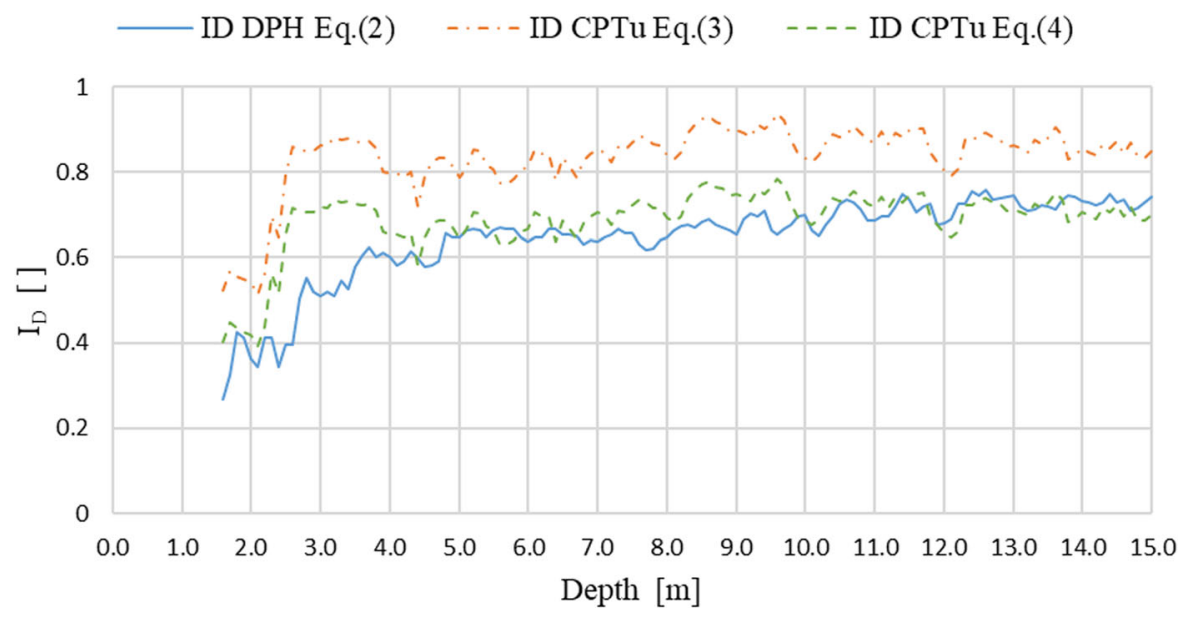

Fig. 4 Variability of density index $I_{D}$ vs depth determined in DPH and CPTu

Table 1 Calculated soil density index values

\begin{tabular}{|c|c|c|c|c|c|c|}
\hline \multirow[t]{2}{*}{ Depth } & \multicolumn{2}{|c|}{$I_{D}$ DPH Eq. (2) [-] } & \multicolumn{2}{|c|}{$I_{D \text { CPTu }}$ Eq. (3) [-] } & \multicolumn{2}{|c|}{$I_{D}$ CPTu Eq. (4) $[-]$} \\
\hline & Mean & $\mathrm{SD}$ & Mean & $\mathrm{SD}$ & Mean & $\mathrm{SD}$ \\
\hline $1.5-3.5 \mathrm{~m}$ & 0.44 & 0.08 & 0.73 & 0.15 & 0.59 & 0.14 \\
\hline $3.5-4.7 \mathrm{~m}$ & 0.60 & 0.01 & 0.81 & 0.04 & 0.67 & 0.04 \\
\hline $4.7-15.0 \mathrm{~m}$ & 0.69 & 0.04 & 0.86 & 0.04 & 0.71 & 0.03 \\
\hline
\end{tabular}

values for all depth ranges, where the greatest $I_{D}$ value was obtained from Eq. (3) based on the CPTu measurement. Very consistent results were also obtained for depths below $4.7 \mathrm{~m}$, from Eqs. (2) and (3) (Fig. 4, Table 1).

Direct comparison of the values recorded in DPH and CPTu tests required using Eq. (5) to find dynamic point resistance $q_{d}$ in the DPH tests, as defined in EN ISO 224676-2.

$q_{d}=\left(\frac{m}{m+m^{\prime}}\right) \cdot r_{d}$

where $m$-hammer mass (for DPH: $50 \mathrm{~kg}$ ); $m^{\prime}-$ total mass of the anvil, hammer guide and rod whose length increases with depth; $r_{d}$-unit dynamic cone resistance.

Unit dynamic cone resistance $r_{d}$, as defined in (7) is determined on the basis of the energy of the hammer blow $\left(E_{\text {theor }}\right)$ in relation to cone area $(A)$ and pile penetration depth per one blow $e$ in (6).

$e=\frac{0.1}{N 10_{H}}$
$r_{d}=\frac{E_{\text {theor }}}{A \cdot e}=\frac{m \cdot g \cdot h}{A \cdot e}$

where $g$-gravitational acceleration; $h$-hammer free falling height.

Figure 5 shows the relationship between depth and the defined values: $e$ - pile penetration depth per one blow (Eq. (6)), $r_{d}$-unit dynamic cone resistance (Eq. (7)) and $q_{d}$-dynamic point resistance (Eq. (5)) for $\mathrm{N1O}_{\text {Hmean }}$ values determined on the basis of four DPH measurements.

\section{Comparative Analysis of Resistances in DPH and CPTu}

This paper focuses on finding $q_{d}$ and $r_{d}$ for coarsegrained soils in order to relate them to static measurements of $q_{c}$ from CPTu.

The results are shown in Fig. 6. Best correlation between $q_{c}$ and $q_{d}$ and between $q_{c}$ and $r_{d}$ is described by the exponential functions provided in the graphs, together with the coefficient of determination $\left(\mathrm{R}^{2}\right)$ at about 0.8 , which allows a satisfactory match between the exponential function and the measurement points. 

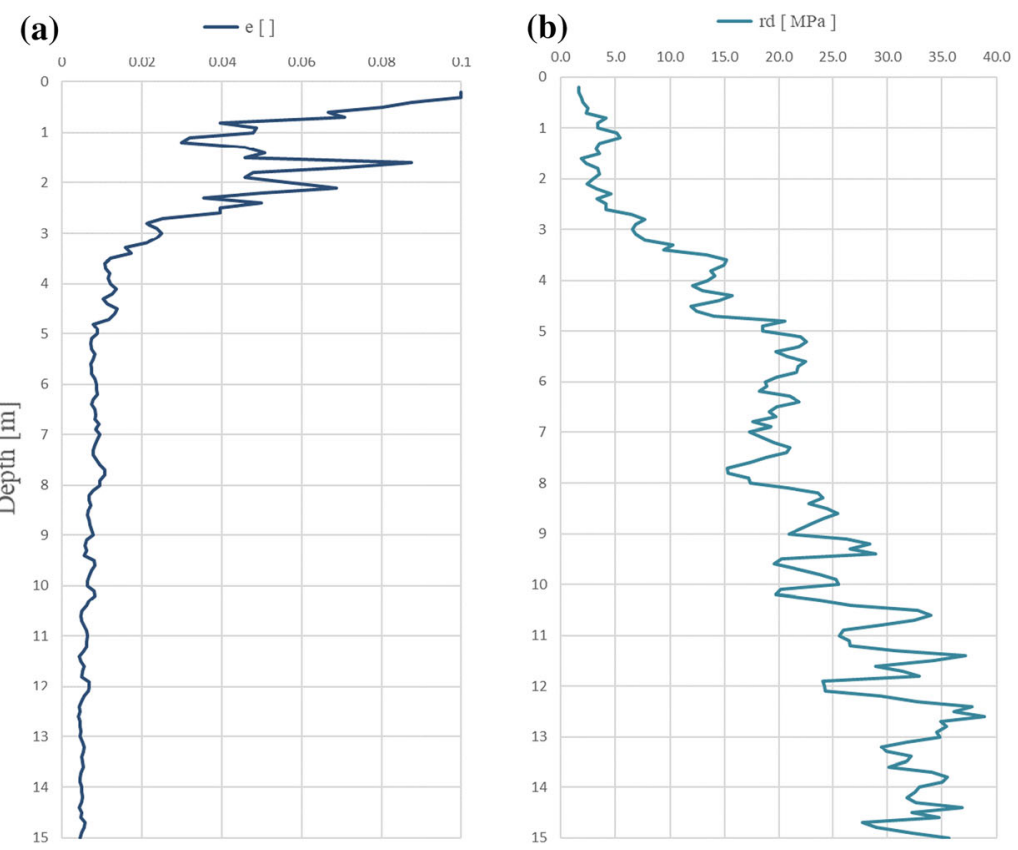

(c)

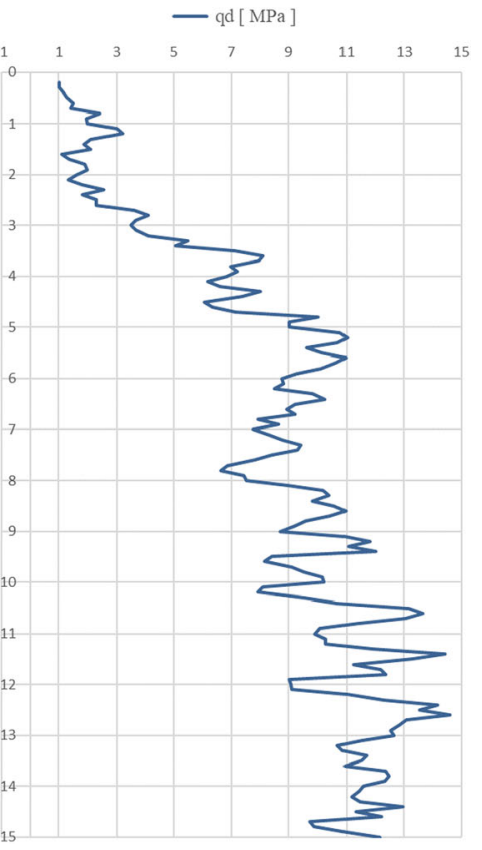

Fig. 5 a Pile penetration depth per one blow (e) Eq. (6); b Unit dynamic cone resistance $\left(r_{d}\right)$ Eq. (7); c Dynamic point resistance $\left(q_{d}\right)$ Eq. (5)

(a)

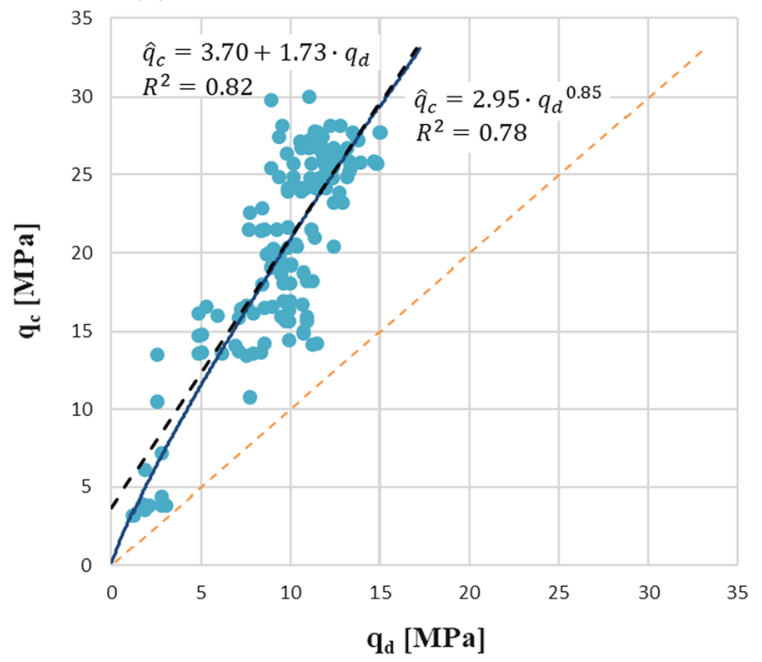

(b)

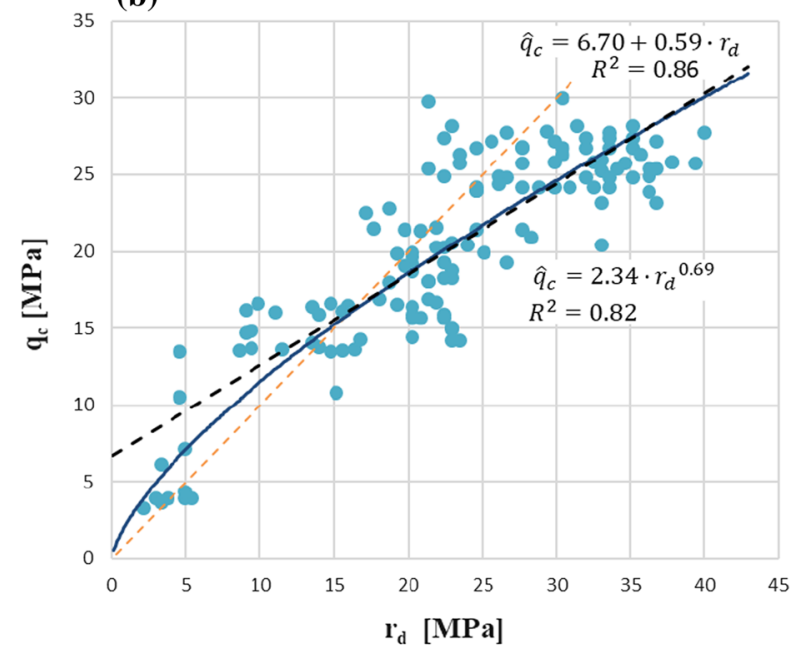

Fig. 6 Results of measured relationships between: a cone resistance $q_{c}$ and dynamic $q_{d}$; b cone resistance $q_{c}$ and unit dynamic resistance $r_{d}$

The Pearson linear correlation coefficient was 0.82 between $q_{c}$ and $q_{d}$ and 0.86 between $q_{c}$ and $r_{d}$. This fact indicates a high positive correlation between the compared values and implies that if for example $q_{d}$ and $r_{d}$ rise, $q_{c}$ should rise as well. In addition, values $q_{d}$ and $r_{d}$ from the DPH test may serve to estimate the $\widehat{q}_{c}$ value (instead of the direct measurement of $q_{c}$ from $\mathrm{CPTu}$, with the use not only of the exponential function (blue continuous line), but also of linear regression functions (black dashed line) provided in Fig. 6a and $b$. 


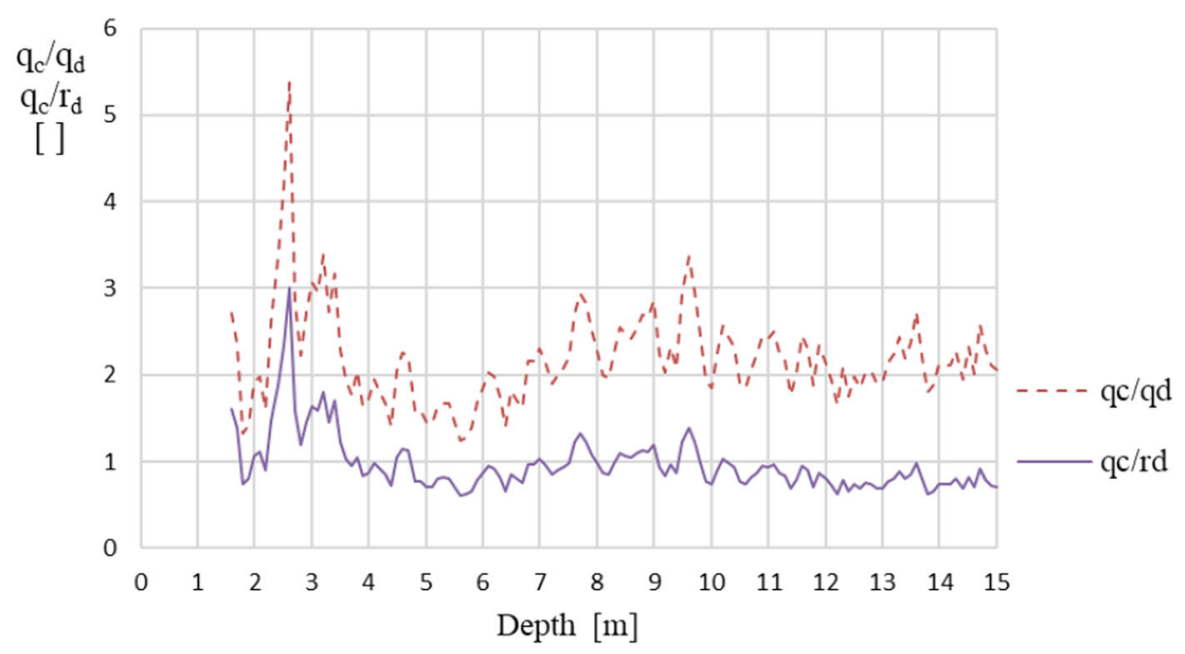

Fig. 7 Relationship between quotients $q_{d} / q_{d}$ and $q_{d} / r_{d}$ as a function of depth

Fig. 8 Correlation between cone resistance $\widehat{q}_{c}$ and the number of blows $N 10_{H}$ based on EN 1997-2:2007 Eurocode 7

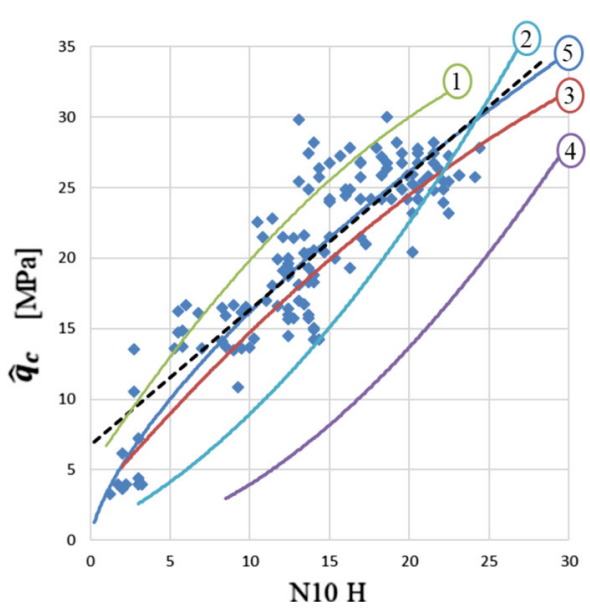

1. Poorly graded sand below the groundwater table

2. Well graded sand and gravel below the groundwater table

3. Poorly graded soil above the groundwater table

4. Well graded sand and gravel above the groundwater table

5. The tested poorly graded sand gravel $(\mathrm{grSa}) /$ medium sand $(M S a)$ below groundwater table $\hat{q}_{c}=3.28 \cdot\left(N_{10 H}\right)^{0.69} ; \mathrm{R}^{2}=0.81$
Comparison of resistances between static and dynamic cones may be represented with quotients $q_{d}$. $q_{d}$ and $q_{c} / r_{d}$ depending on the measurement depth (Fig. 7). In a lithologically uniform soil, DPH and $\mathrm{CPTu}$ tests must overcome resistances related to effective stress which increases with depth. This effect can be seen more clearly in the $q_{d}$ and $r_{d}$ graph (Fig. 5 b, c) than in the $q_{c}$ graph (Fig. 1). In this case (Fig. 7), an observation can be made that below $3.5 \mathrm{~m}$ b.g.l., where the soil is much more uniform, the quotient values have lower variability than above $3.5 \mathrm{~m}$ b.g.l. Mean value of $q_{d} / q_{d}$, below $3.5 \mathrm{~m}$ b.g.l. becomes stable at 2.10 with standard deviation $\mathrm{SD}=0.39$ and coefficient of variability $\mathrm{CV}=18.6 \%$, while $q_{d} / r_{d}$ is 0.88 as $\mathrm{SD}=0.16$ and $\mathrm{CV}=18.2 \%$. The results here presented correspond to the results presented in other works, in which $q_{d} / q_{d}$ for coarsegrained soils was obtained at 2.3 (Gadeikis et al. 2010) and 1.85 (Czado and Pietras 2012).

\section{Discussion of Results}

Eurocode 7 allows the possibility of estimating $\widehat{q}_{c}$ on the basis of DPH tests. The standard includes a nomograph which proves very helpful in estimating $\widehat{q}_{c}$ on the basis of $N 1 O_{H}$, as presented in Fig. 8 .

Figure 8 shows curves 1, 2, 3 and 4 which represent proposals taken from Eurocode 7 for water-saturated and water-unsaturated soils, well and poorly graded 
sands and sand/gravels. The original results show measurement points from mean $q_{c}$ values read every $10 \mathrm{~cm}(\mathrm{CPTu})$ and the corresponding mean measurement values $\mathrm{N1O}_{\text {Hmean }}$ read from the four DPH tests at the same depth. The curve No 5 is a representation of an exponential function which best describes the correlation between the considered values of $q_{c}$ and N10 Hmean. The curve obtained in this research is similar to curves 1 and 3 which describe poorly graded soils being sands below and above the groundwater table.

In order to emphasize the correlation between $q_{c}$ and $N 10_{\text {Hmean }}$, the Pearson linear correlation coefficient was determined, which in this case was 0.86 for linear regression function having the form of Eq. (8). This function is represented with the dashed line in Fig. 8.

$\hat{q}_{c}=6.70+0.96 \cdot N 10_{H}$

\section{Conclusions}

The test results presented in this work are in accordance with the nomograph of Eurocode 7 (Fig. 8), which is suggested for estimating cone penetration $\left(\widehat{q}_{c}\right)$ in sands and sand-gravels on the basis of the DPH dynamic tests.

A positive verification, as presented in the nomograph (Fig. 8), allows (in the discussed area) using the proposed empirical correlations between $\mathrm{N} 10_{\mathrm{H}}$ and $\widehat{q}_{c}$ (Fig. 8). The $\widehat{q}_{c}$ values calculated directly from the DPH tests may be used to find pile capacity base (Ćosić et al. 2016; Wyjadłowski et al. 2018; Baca et al. 2016). In other geotechnical conditions, the method should be used with precautions. The correlation which allows estimating cone resistances $\widehat{q}_{c}$ was determined for poorly graded, coarsegrained soils (SBT6, sand-gravels $(\mathrm{grSa}) /$ medium sands $(M S a)$ ) below groundwater table, based on four DPH tests and one CPTu test. Such approach is occasionally necessary when direct CPTu probing is rendered difficult or impossible by e.g. insufficient test site area, no possibility to anchor the CPTu probe or significant resistances $q_{c}>25 \mathrm{MPa}$.

The obtained correlations between $q_{c}$ and $q_{d}$ as well as between $q_{c}$ and $r_{d}$ were determined on a limited base of tests (Fig. 6). However, the calculated functions have the coefficient of determination $\left(\mathrm{R}^{2}\right)$ at a level of approx. 0.8 , showing a satisfactory match level between the functions and the measured values. The Pearson linear correlation coefficient at 0.8 also indicates a high positive correlation between the compared values and implies that if for example $q_{d}$ and $r_{d}$ rise, $q_{c}$ should rise as well.

The calculated quotient of $q_{c}$ over $q_{d}$ at 2.10 with $\mathrm{SD}=0.39$ (Fig. 7) correspond to the results presented in other works, in which qc/qd for coarsegrained soils was obtained at 2.3 (Gadeikis et al. 2010) and 1.85 (Czado and Pietras 2012). This fact confirms that it is possible to estimate the $\widehat{q}_{c}$ values on the basis of $q_{d}$ from the DPH test.

The results of correlations between values measured in DPH and CPTu tests, as presented in this work, allow considering the possibility to use the DPH tests as an alternative to the CPTu tests, especially in the case of coarse-grained soils in which static probing is hindered by great penetration resistance.

Open Access This article is licensed under a Creative Commons Attribution 4.0 International License, which permits use, sharing, adaptation, distribution and reproduction in any medium or format, as long as you give appropriate credit to the original author(s) and the source, provide a link to the Creative Commons licence, and indicate if changes were made. The images or other third party material in this article are included in the article's Creative Commons licence, unless indicated otherwise in a credit line to the material. If material is not included in the article's Creative Commons licence and your intended use is not permitted by statutory regulation or exceeds the permitted use, you will need to obtain permission directly from the copyright holder. To view a copy of this licence, visit http://creativecommons.org/licenses/by/4.0/.

\section{References}

Ampadu SIK, Ayeh FF, Boadu F (2018) Deriving SPT N-values from DCP test results: the case of foundation design in a tropical environment. Geotech Geol Eng 36(4):2517-2531

Baca M, Rybak J, Tamrazyan AG, Zyrek T (2016) Pile foot capacity testing in various cases of pile shaft displacement. Int Multidiscip Sci GeoConf SGEM Surv Geol Min Ecol Manag 1:945-950

Bałachowski L (2006) Penetration resistance of Lubiatowo sand in calibration chamber tests. Arch Hydro Eng Environ Mech 53(4):311-329

Bustamante M., Gianeselli L (1993) Design of auger displacement piles from in situ tests. Proc. 2nd Inter. Geotechnical Seminar on Deep Foundations on Bored and Auger Piles. (Ghent, Belgium). A.A. Balkema. Rotterdam 
Ćosić M, Šušić N, Folić R, Bancila R (2016) Probabilistic analysis of bearing capacity of piles with variable parameters in CPT Test and Calculation according to the requirements of eurocode 7 (en 1997-1:2004) regulations. Struct Integr Life 16(1):25-34

Czado B, Pietras JS (2012) Comparison of the cone penetration resistance obtained in static and dynamic field tests. AGH J Min Geoeng 36(1):97-105

dos Santos MD, Bicalho KV (2017) Proposals of SPT-CPT and DPL-CPT correlations for sandy soils in Brazil. J Rock Mech Geotech Eng 9(6):1152-1158

EN 1997-2:2007 Eurocode 7. Geotechnical design. Ground investigation and testing

EN ISO 14688-1:2018 Geotechnical investigation and testingIdentification and classification of soil-Part 1: Identification and description

EN ISO 14688-2:2018 Geotechnical investigation and testing-Identification and classification of soil-Part 2: Principles for a classification

EN ISO 17892-4:2016 Geotechnical investigation and testingLaboratory testing of soil-Part 4: Determination of particle size distribution

EN ISO 22476-1: 2012 Geotechnical investigation and testingField testing-Part 1: Electrical cone and piezocone penetration test

EN ISO 22476-2:2005 Geotechnical investigation and testingField testing-Part 2: Dynamic probing

Gadeikis S, Žaržojus G, Urbaitis D (2010) Comparing CPT and DPSH in Lithuanian soils, 2and International Symposium on Cone Penetration Testing, Huntington Beach, CA, USA. Volume 2\&3: Technical Papers, Session 3: Applications: 3-22

Jamiolkowski M, Lo Presti DCF, Manassero M (2003) Evaluation of relative density and shear strength of sands from CPT and DMT. Soil Behav Soft Gr Constr 119:201-238
Kodicherla SPK, Nandyala DK (2016) Use of CPT and DCP based correlations in characterization of subgrade of a highway in Southern Ethiopia Region. Int J Geo Eng 7(1):11

Lingwanda MI, Larsson S, Nyaoro DL (2015) Correlations of SPT, CPT and DPL data for sandy soil in Tanzania. Geotech Geol Eng 33(5):1221-1233

Lunne T, Robertson PK, Powell JJM (1997) Cone penetration testing in geotechnical practice

Mahler A, Szendefy J (2009) Estimation of CPT resistance based on DPH results. Period Polytech Civ Eng 53(2):101-106

Melzer K-J (1968) Sondenuntersuchungen in Sand Mitt. Inst. f Verkehrsbau, Grundbau und Bodenmechanik an der $\mathrm{TH}$ Aachen, No 43

Pinheiro C, Molina-Gómez F, Rios S, da Fonseca AV, Miranda $\mathrm{T}$ (2018) Correlations between Dynamic Penetrometer Light and Cone Penetration Tests in Intermediate Soils: a Statistical Comparison. XIX Congresso Brasileiro de Mecânica dos Solos e Engenharia Geotécnica Geotecnia e Desenvolvimento Urbano

Robertson PK (2010) Soil behaviour type from the CPT: an update. In: 2nd international symposium on cone penetration testing, USA, 9-11 May 2010

Stenzel G, Melzer KJ (1978) Soil investigations by penetration testing according to DIN 4094. Tiefbau 20:155-160

Wyjadłowski M, Bagińska I, Rainer J (2018) Probabilistic assessment of pile capacity based on CPTu probing including random pile foundation depth. In: MATEC Web of Conferences (Vol 196, p 01058). EDP Sciences

Publisher's Note Springer Nature remains neutral with regard to jurisdictional claims in published maps and institutional affiliations. 